\title{
OPTICAL CHARACTERIZATION OF CdZnSe/ZnSe MULTIQUANTUM WELL SYSTEM
}

\author{
M. GodLEWSKI, K. KaRPIŃSKA \\ Institute of Physics, Polish Academy of Sciences \\ Al. Lotników 32/46, 02-668 Warszawa, Poland \\ J.P. Bergman, B. Monemar
}

Department of Physics and Measurement Technology, Linköping University 58183 Linköping, Sweden

E. KURTZ AND D. HOMMEL

Institute of Physics, Würzburg University, Am Hubland, 8700 Würzburg, Germany

Optical properties of $\mathrm{Cd}_{x} \mathrm{Zn}_{1-x} \mathrm{Se} / \mathrm{ZnSe}(x=0.12)$ multiquantum well system are discussed. The transient photoluminescence and optically detected cyclotron resonance experiments demonstrate a strong contribution of bound exciton emission to the low temperature photoluminescence spectra.

PACS numbers: 68.60.-p, 71.35.+z, 76.70.Hb

\section{Introduction}

The CdZnSe/ZnSe multiquantum well (MQW) and superlattice structures are currently intensively studied due to their possible application in blue light emitting and semiconductor laser diodes [1]. In this communication we present the results of photoluminescence (PL) transient and optically detected cyclotron resonance (ODCR) experiments. The aim of the present study is to identify recombination transitions from the QWs and to describe their properties. We show that bound exciton spectra contribute to the low temperature PL emission from the QWs. 


\section{Experimental results and discussion}

In Fig. 1 we show a PL spectrum from the CdZnSe/ZnSe MQW sample. The MQW structure studied consisted of four CdZnSe ( $12 \%$ of $\mathrm{Cd}$ ) quantum wells of 114, 76, 43 and $22 \AA$ thickness separated by $575 \AA$ ZnSe barriers. They were grown by molecular beam epitaxy (MBE) on a GaAs substrate covered with a $3900 \AA \mathrm{ZnSe}$ buffer layer and with a $325 \AA$ cap layer on top of the structure. The PL spectrum, shown in Fig. 1, consists of four emission bands, related to the QWs with a width of $22 \AA, 43 \AA, 76 \AA$ and $114 \AA$ (from left to right). The PL emission from the $22 \AA \mathrm{QW}$ has two components separated by $10 \AA$. The temperature dependence of the PL intensity was studied. We observe a strong temperature dependence of the PL spectrum intensity and of its spectral position. At increased temperatures the intensity of the low energy component of the QWs PL decreases more rapidly. At higher temperatures it could be observed that the PL emission from the $43 \AA \mathrm{QW}$ also has two components, with a higher energy component (attributed to localized exciton) being weak at $2 \mathrm{~K}$ temperature and becoming stronger at increased temperature. Emission intensity is anticorrelated with the QWs thickness, i.e. the decrease is more rapid for narrower QWs. The red shift of the exciton emissions (by about $10 \AA$ ) at increased temperatures $(100 \mathrm{~K})$ can be explained by the temperature dependence of the fundamental band gap. We speculate that also the misfit strain is temperature dependent and that this to some extent can contribute to the observed shift.

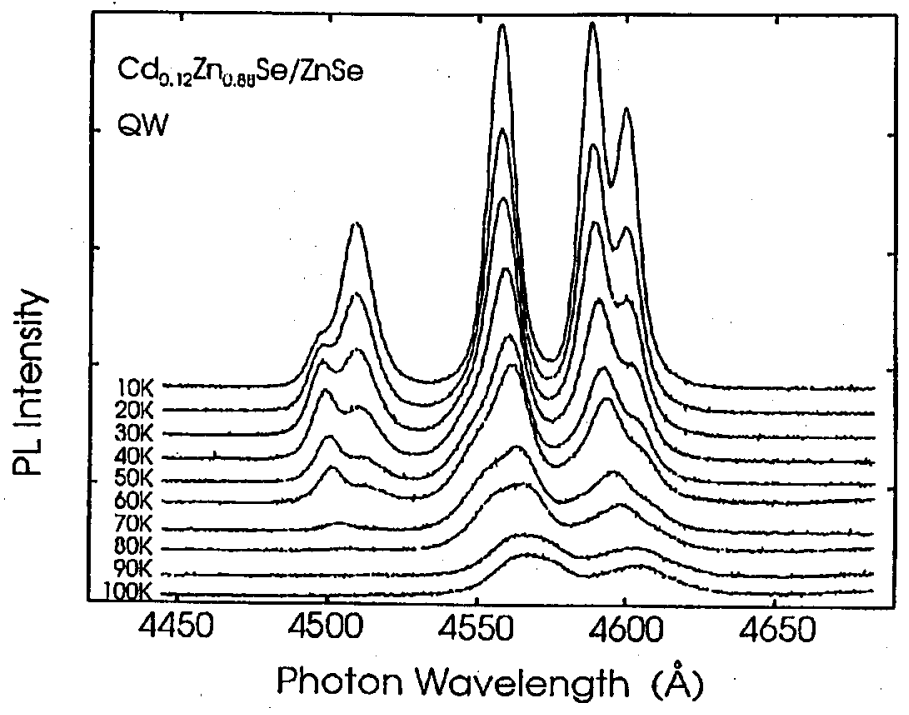

Fig. 1. Photoluminescence spectrum of $\mathrm{CdZnSe/ZnSe} \mathrm{MQW} \mathrm{system.} \mathrm{The} \mathrm{PL} \mathrm{emission}$ observed comes from $22 \AA, 43 \AA, 76 \AA$ and $114 \AA \mathrm{QWs}$ (from left to right). The double structure observed for the $22 \AA$ and (at increased temperature) for the $43 \AA \mathrm{QW} \mathrm{PL}$ emission is due to simultaneous observation of free and bound exciton PL emissions. 
We also performed time resolved measurements of the different emissions with a streak camera system. The time resolved kinetics from the $22 \AA, 76 \AA$ and $114 \AA \mathrm{QW}$ were almost identical, with the high energy component having a decay time of $76 \mathrm{ps}$ and the lower energy component a decay of $85 \mathrm{ps}$ and a slightly longer rise time. It is interesting to notice that the dominant PL emission at $2 \mathrm{~K}$ from the $43 \AA \mathrm{QW}$ has a shorter decay time $(72 \mathrm{ps})$. than the lower energy PL band observed from the $22 \AA \mathrm{QW}$ and the $76 \AA$ and $114 \AA \mathrm{QW}$. We relate this fact to some energy transfer between excitonic transitions in the QWs. The PL transitions do not change their spectral position during the time of the decay. This is in contrast to the significant shift observed in e.g. narrow GaAs/AlGaAs QWs [2]. This indicates that the excitons do not diffuse between localized states and that they therefore are strongly localized excitons. The width of the excitonic transitions (about $3.5 \mathrm{meV}$ ) reflects the magnitude of potential fluctuations present in the structure studied.

We attribute the lower energy component of the $22 \AA \mathrm{QW}$ emission and the dominant PL band from the $43 \AA \mathrm{QW}$ to a recombination of the bound exciton (BE) (neutral donor bound exciton). The identity of defect binding an exciton remains unknown since our structures were not intentionally doped. There are several arguments for such an identification of these PL bands. First is their strong temperature dependence. The emission decreases in intensity with increasing temperature and $\mathrm{BE}$ are not observed at higher temperatures. This is expected due to thermal ionization of BE. They have both longer rise and decay time, which is a consequence of the fact that $\mathrm{BE}$ is created by capture of a free exciton (FE). The latter explains why a shorter decay time is observed for the $43 \AA \mathrm{QW}$ at $2 \mathrm{~K}$. In this case the contribution from the localized exciton was very weak and the energy transfer link between localized and bound exciton was stronger resulting in shorter decay of the bound exciton.

In Fig. 2 we show the spectral dependence of the ODCR signal (ODCR-PL), which represents the PL changes caused by hot carriers heated at CR conditions (hea vily damped CR resonance was observed). The ODCR-PL spectrum was measured in phase with on-off modulated microwaves. $1 \mathrm{kHz}$ frequency of microwave modulation was used to minimize lattice heating effects. The ODCR-PL spectrum shows a typical response of the PL consisting of two competing recombination channels - free and bound excitons. At increased microwave power bound excitons are impact ionized by collisions with hot carriers, which enhances recombination via a free exciton channel [3]. It is interesting to notice that the ODCR-PL experiment clearly shows the two band character of the $43 \AA \mathrm{QW}$ emission, even though it was not resolved in the low temperature PL experiment and was only partly resolved in the transient PL study. Such increased spectral resolution of the ODCR-PL experiment was reported previously from our study of GaInAs [4]. The main effect of carrier heating at $\mathrm{CR}$ conditions is an intensity redistribution between the two excitonic transitions. We did not observe diffusion of hot carriers out of the QWs, the effect dominant for the CdTe/CdMnTe MQW structures [5]. 


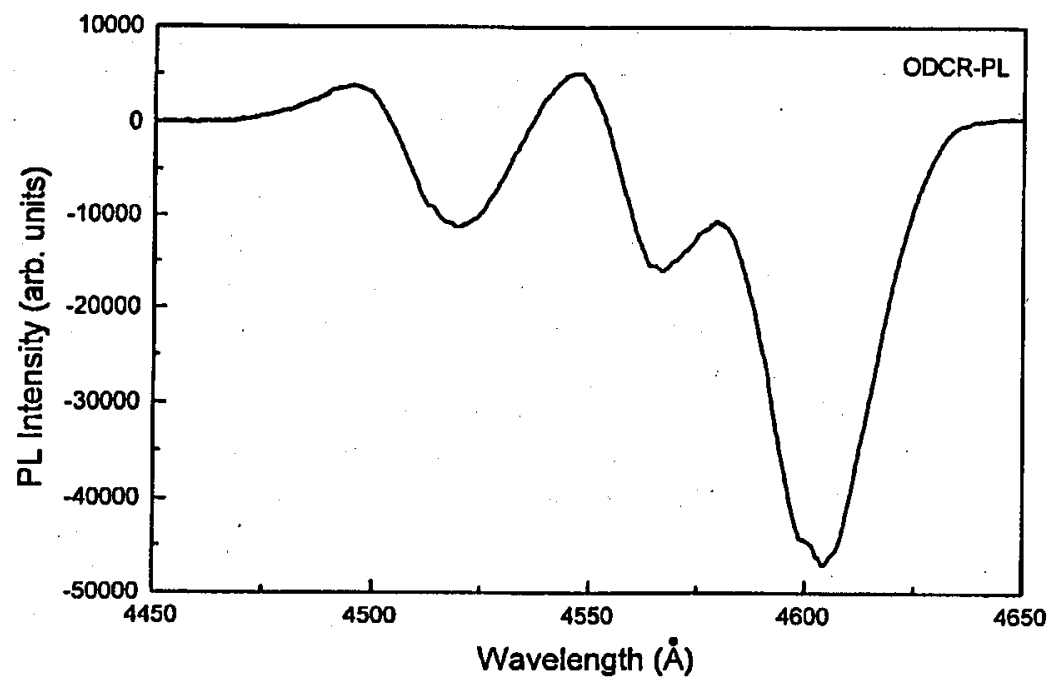

Fig. 2. The spectral dependence of the ODCR signal (ODCR-PL) of CdZnSe/ZnSe MQW system. The ODCR-PL spectrum was measured in phase with on-off modulated microwaves at $1 \mathrm{kHz}$ and magnetic field set at damped $\mathrm{CR}$ signal.

\section{References}

[1] M.A. Haase, J. Qin, J.M. DePuydt, M. Chen, Appl. Phys. Lett. 59, 1272 (1991).

[2] M. Zachau, J.A. Kash, W.T. Masselink, Phys. Rev. B 44, 8403 (1991).

[3] H. Weman, M. Godlewski, B. Monemar, Phys. Rev. B 38, 12525 (1988).

[4] M. Godlewski, K. Fronc, M. Gajewska, W.M. Chen, B. Monemar, Phys. Rev. B 44, 8357 (1991).

[5] M. Godlewski, K. Świątek, C.I. Harris, J.P. Bergman, B. Monemar, A. Waag, Acta Phys. Pol. A 87, 213 (1995). 